\title{
A Rare Case of Ossified Chronic Subdural Hematoma Complicated with Tension Pneumocephalus
}

\author{
Mehmet Turgut $^{1}$ Murat Ö. Yay ${ }^{1}$ \\ ${ }^{1}$ Department of Neurosurgery, Faculty of Medicine, Aydın Adnan \\ Menderes University, Efeler/Aydın, Turkey \\ J Neurol Surg Rep 2019;80:e44-e45.

\begin{abstract}
Address for correspondence Mehmet Turgut, MD, PhD, Department of Neurosurgery, Faculty of Medicine, Aydın Adnan Menderes University, Efeler/Aydın 09010, Turkey
\end{abstract} \\ (e-mail: drmturgut@yahoo.com).
}
Abstract
Keywords
- chronic subdural hematoma
- postoperative complication
- tension pneumocephalus

\begin{abstract}
A 59-year-old man presented with epileptic seizures interpreted as episodic syncope in the past 3 years and the patient had a history of head trauma about 4 years ago. Computed tomography revealed an ossified chronic subdural hematoma involving the right frontotemporoparietal region, which was totally resected using microsurgical technique. Postoperatively, weakness developed in right arm and magnetic resonance imaging revealed a bilateral tension pneumocephalus, which was immediately treated by a left frontal burr hole trepanation, and the patient was discharged uneventfully.
\end{abstract}

\section{Introduction}

Calcification or ossification is observed at a rate of 0.8 to $10 \%$ in patients with chronic subdural hematoma (CSDH). ${ }^{1,2}$ Until recently, less than 100 cases of calcified or ossified CSDH have been published in the English literature. Herein, we present such a case in which tension pneumocephalus developed following microsurgical excision of the ossified lesion involving the right frontotemporoparietal region.

\section{Case Report}

A 59-year-old male patient presented with the epileptic seizures interpreted as episodic syncope that had been persisting for 3 years, once or twice in a year. The patient had a history of head injury about 4 years ago. Neurological examination was normal with exception of left hemihypoesthesia. A preoperative computed tomography (CT) scan demonstrated a large mixed-density CSDH lesion bordered by a hyperdense rim in the right frontotemporoparietal region, causing displacement of the right lateral ventricle (-Fig. 1A). During the surgical procedure, a right frontotemporoparietal craniotomy was done and the dura mater with ossified hard external capsule was opened following lifting the bone ( - Fig. 1B). After the fluid was completely drained, the ossified internal capsule of CSDH surrounding the cerebral cortex, which had the appearance of an eggshell, was exposed and excised by a careful dissection from the arachnoid mater using microsurgical techniques (-Fig. 1C). Pathology suggested the diagnosis of ossified CSDH. On postoperative third day, the patient developed weakness in right arm and drowsiness; magnetic resonance imaging revealed complete removal of the hematoma, but there was tension pneumocephalus in both frontal regions with significant mass effect, called Mount Fuji sign in CT scan (-Fig. 1D, E). Therefore, a right frontal burr hole trepanation was immediately performed and tension pneumocephalus treated successfully. A repeat CT scan at 30 days showed disappearance of the mass and pressure effect ( $\mathbf{F i g . ~ 1 F ) . ~}$

\section{Discussion}

Although the mechanism of its development is unclear, it has been suggested that development of an ossified CSDH generally takes a few years. ${ }^{1,2}$ Clinically, headache and seizure are the most frequent symptoms, but the majority of the cases are asymptomatic. In clinical practice, surgical excision of the calcification is not routinely performed in asymptomatic and old patients, because of the risk of damaging the underlying cortex and they are followed up. ${ }^{1}$ On the contrary, tension pneumocephalus, which may cause the Mount Fuji sign or
License terms

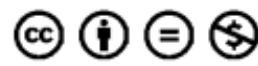

received

January 15, 2019

accepted

June 16, 2019
DOI https://doi.org/

10.1055/s-0039-1694738. ISSN 2193-6358. (c) 2019 Georg Thieme Verlag KG Stuttgart · New York 

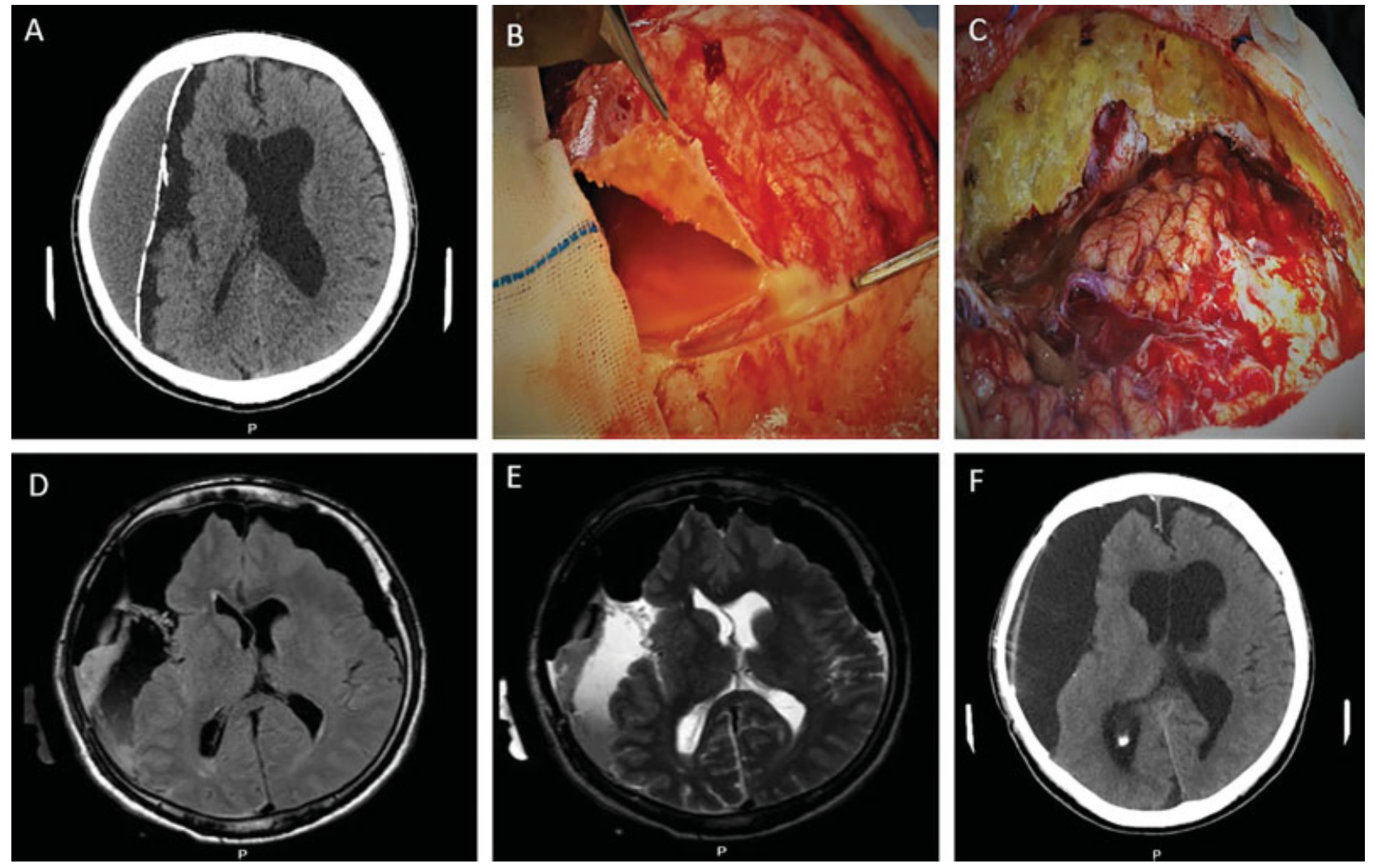

Fig. 1 (A) CT scan showing a large subdural collection. (B, C) Photograph of a muddy white material when dura mater with ossified external capsule of CSDH was open and partial excision of the ossified internal capsule from the cerebral cortex. (D, E) T1- and T2-weighted sagittal MRIs demonstrating compression of both frontal lobes by subdural hypertensive pneumocephalus, similar to the silhouette of Mount Fuji. (F) A repeat CTscan 1 month later showed a radiologic improvement in the mass effect. CSDH, chronic subdural hematoma; CT, computed tomography; MRI, magnetic resonance imaging.

peaking sign as intracranial subdural air collections causing bilateral compression of the frontal lobes, is an uncommon and life-threatening neurological condition. ${ }^{3,4}$ The incidence of tension pneumocephalus developing after the evacuation of a CSDH ranged 0 to $16 \%$, and it may be fatal owing to brain herniation if tension pneumocephalus is not diagnosed early and treated properly via decompression of the cranial cavity by a burr hole or needle aspiration. ${ }^{3,4}$ To prevent the development of pneumocephalus after surgery for calcified or ossified CSDH, it is useful to avoid the following contributing factors: meningeal defect, hydrocephalus, nitrous oxide anesthesia, osmotherapy, hyperventilation, and infections. ${ }^{4}$

This case highlights the importance of considering tension pneumocephalus as a serious postoperative complication in patients with calcified or ossified CSDHs. To the best of our knowledge, a similar case of CSDH complicated with postoperative tension pneumocephalus, called Mount Fuji sign in
CT scan as an indicator of a neurosurgical emergency has not been reported in the literature to date.

\section{Conflict of Interest}

None.

\section{References}

1 Turgut M, Palaoğlu S, Sağlam S. Huge ossified crust-like subdural hematoma covering the hemisphere and causing acute signs of increased intracranial pressure. Childs Nerv Syst 1997;13(07): 415-417

2 Turgut M, Samancoğlu H, Ozsunar Y, Erkuş M. Ossified chronic subdural hematoma. Cent Eur Neurosurg 2010;71(03):146-148

3 Tsai CC, Chao CM. Tension pneumocephalus with Mount Fuji sign. Intern Med 2017;7:3

4 Sanyal P, Bele K, Vishwanath Rai SP. Tension pneumocephalus: Mount Fuji sign. Arch Med Health Sci 2015;3:352-353 